\title{
Carbon TEM grids fabricated using C-MEMS as the platform for suspended carbon nanowire characterization
}

Bidhan Pramanick $^{1,2}$, Arnoldo Salazar ${ }^{2}$, Sergio O. Martinez-Chapa*, ${ }^{* 1}$, Marc J. Madou ${ }^{*, 1,2}$ 


\begin{abstract}
Carbon transmission electron microscope (TEM) grids were fabricated for the first time using Carbon-MEMS (C-MEMS) process. The C-MEMS process and its application in the fabrication of carbon TEM grids are detailed. The benefits of making carbon TEM grids this way are also explained. Commercially available TEM grids are often hard to use for many types of samples. Moreover sample preparation such as nanowire placement on conventional TEM grids remains very challenging. Here we demonstrate a novel fabrication method of carbon TEM grids. These grids serve as a support for the nanowire deposition process itself; no further nanowire placement is required. The method for constructing in-situ carbon TEM grids is illustrated for the study of suspended carbon nanowires. The method is generic as long as the nano-devices that are being built can survive the pyrolysis step. In this paper, a test-bed for CNW TEM study is fabricated using one step photolithography to pattern SU8 TEM grids and then fibers are electrospun over the grid. Both, grids and fibers are then converted to carbon at the same time by pyrolysis in an inert $\left(\mathrm{N}_{2}\right)$ environment. The grids are made on a $\mathrm{Si} / \mathrm{SiO} \mathrm{O}_{2}$ substrate and are released from the substrate using Buffered Oxide Etch.
\end{abstract}




\section{Introduction}

Carbon constitutes the most suitable choice for replacing silicon in many micro- and nano-fabrication developments. Si-based technology still dominates today as it is the starting material for the fabrication of ICs which made silicon the most prominent technological material for over 50 years. However, silicon technology often limits developments in bio-sensors [1,2], gas sensors [3], nano-electronics [4], micro-batteries [5], etc, mainly because of the difficulties in three dimensional structuring and unavoidable substrate interference. Silicon also remains the dominant material in micro- and nanoelectro-mechanical systems (MEMS and NEMS), however in recent years MEMS and NEMS based on carbon (C-MEMS and C-NEMS) have emerged as a viable alternative to overcome some of the drawbacks of silicon.

A major benefit of carbon over $\mathrm{Si}$ is that it is available in various allotropes such as diamond, graphite, carbon nanotubes (CNTs), glassy carbon, each with unique physicochemical properties [6]. Three dimensional mechanical machining of most of carbon into micro- or nano-devices is not straightforward, as the material is usually hard and brittle. Focused ion beam (FIB) milling [7] and reactive ion etching (RIE) [8] are more up to the task but these are time consuming and very expensive. Low feature resolution and poor repeatability of the carbon composition limit the application of the less expensive screenprinting technique for the fabrication of carbon devices [2]. In sharp contrast, polymers can be molded easily and then converted to carbon through pyrolysis. In other words the underlying principle of C-MEMS/C-NEMS is to choose an easy to work with polymer precursor, mold or photo pattern this precursor material (in the case of a photo resist), and then convert it to carbon by pyrolysis $[2,6,9]$. 
The C-MEMS/C-NEMS [10] process is illustrated in Figure 1 and includes: spin coating of a carbon precursor photoresist, soft bake (not shown), UV exposure, post bake (also not shown), and development [6]. After photolithography, carbon structures are obtained through a pyrolysis process. The latter is carried out in an open-ended quartz-tube furnace with an inert $\mathrm{N}_{2}$ atmosphere, in which samples are first heated at $300^{\circ} \mathrm{C}$ for about $60 \mathrm{~min}$ and then heated again at $900^{\circ} \mathrm{C}$ for $60 \mathrm{~min}$. In the C-MEMS/ C-NEMS process that we used to fabricate the carbon transmission electron microscope (TEM) grids, a photoresist is patterned by lithography and pyrolyzed at high temperatures in an oxygenfree environment. By changing the lithography conditions, soft and hard baking times and temperatures, resist additives (e.g., a catalyst), pyrolysis time, temperature, and environment, the C-MEMS/C-NEMS process permits a wide variety of interesting new applications that employ structures having a wide variety of shapes, resistivities, mechanical and other physico-chemical properties.

The resulting carbon material microstructure for the process detailed above was found to be that of glassy carbon [10,11]. Glassy carbon has excellent electrochemical properties and is better for most electrochemical applications than noble metals like Pt and $\mathrm{Au}$ which are commonly used for miniaturized sensing electrodes [12,13]. Besides electrochemical sensors, some other examples of C-MEMS/C-NEMS applications reported in the literature include: batteries [5], fuel cells [14], electrodes for dielectrophoresis [15], supercapacitors [16], biological scaffolds [17], hot nanowires for local CVD [18], and gas sensors [19].

Carbon nanotubes (CNTs) and carbon nanofibers (CNFs) feature large surface to volume ratios that make them unique for various sensor applications. Particularly CNFs 
that are easier to fabricate and control than CNTs, have numerous applications ranging from sensors to nano-electronics (interconnects and active devices) [20,21]. Because nanowires have a high surface to volume ratio, surface charge changes caused by the sensing phenomenon have a larger effect on the overall resistivity/impedance than in the case of bulk wires and this results in an improved lower limit of detection (LOD) [22]. Suspended carbon nanowires feature several further advantages. Suspended nanowires are removed from the substrate and this avoids deleterious substrate influences such as contamination and surface charge shunting. In addition, the LOD is also improved because of higher mass transport due to non-linear diffusion effects and because of the wire can be approached by analyte molecules from all directions. By derivatizing the carbon nanowire surface with a monolayer of sensor molecules, say protein or DNA molecules, the nanowires can be turned into nano-immuno or nano-DNA sensors by monitoring how the binding of the complementary molecules modulates the nanowires' impedance. When coating the carbon nanowires (CNWs) with metal oxide semiconductors (MOS) they can also be turned into nanogas sensors [22]. The fabrication process of these suspended carbon nanowires involves a combination of photo-patterning of SU8 for making supporting structures, and electro mechanical spinning (EMS) [23] for depositing the suspended nanowires. SU8 is a negative photoresist consisting of bisphenol, a novolac epoxy resin, dissolved on gamma-butyrolactone, GBL or cyclopentanone, and a photoinitiator that composes $10 \%$ wt of the resin mass [24]. The chemical structure of SU8 is shown in Figure 2a [25] and a hybrid microstructure construct is illustrated in Figure $2 \mathrm{~b}$ and 2c where we show a set of carbonized EMS fibers suspended over a carbon support structure.

\section{Transmission Electron Microscopy of CNWs}


TEM is a well-established technique where samples are prepared on commercially available grids (e.g. $\mathrm{Cu}$ grids) as sample supports and mounted on the TEM specimen holder. However, obtaining specimens of the right thickness is one of the most challenging aspects in TEM analysis. The maximum allowable thickness depends on the electron scattering factor of the material studied. As a general rule the higher the atomic number of the elements in the sample, the greater the electron scattering factor and the thinner the specimen needs to be made [26]. For example, using 100-KV electrons in the case of Aluminum $(\mathrm{Al})$ requires the sample thickness to be in the range of a few hundred nanometers whereas for Uranium (U) the useable thickness range is a few tens of nanometers [26].Various specimen preparation techniques are used for thinning samples that are too thick for TEM analysis. Thinning might involve polishing, ion milling, ultrasonic drilling, chemical micromachining, jet drilling FIB/SEM preparation, etc. Generally, the samples are machined to their allowable thickness before placing them on the grids. However, very small/thin specimens are very difficult to place on the TEM grids and therefore further thinning is often performed after placing the specimen on the grid. As a consequence, the TEM grid used needs to be able to withstand high temperatures and be chemical resistant, etc. The C-MEMS TEM grids, we have developed here, can accomplish all these requirements.

Perhaps an even bigger challenge than thinning the sample is placing the thinned nano structures onto the TEM grid. Based on the nanoparticle surface charge, the grid surface often needs to be functionalized for better attachment of the nanoparticles to the grid. TEM sample grids with a continuous silicon oxide film, that is electron transparent, are used for negatively charged nanoparticles. Derivatization of the grid surface by 
exposing it to a small amount of amino-propyl-dimethyl-ethoxy-silane [APDMES, $\mathrm{NH}_{2}-$ $\left.\left(\mathrm{CH}_{2}\right)_{3}-\mathrm{Si}-\left(\mathrm{CH}_{3}\right)_{2}\left(\mathrm{C}_{2} \mathrm{H}_{5} \mathrm{O}\right)\right]$ solution positively charges the grid surface which then attract and bind to negatively charged nanoparticles [27]. For the neutrals and positively charged particles, one of the approaches is by mixing the particle suspension with a dilute solution of a suitable macromolecular agent, like Bovine serum albumin (BSA) which is cheap, widely available and well-studied, at the appropriate concentration ratio [28]. The approach relies on the stabilization of individual particles against aggregation and mitigating dewetting. There is also a common method, called glow discharge technique, used to modify the grid surface into a more hydrophilic one for the better attachment of samples, especially biological samples, to the grid [29]. Traditionally nanoparticles placement is accomplished either by drop-casting a nanoparticle dispersion onto the grid or by dipping the grid into the nanoparticle dispersion. The dispersion solution is then removed from the grid surface by wicking with a filter paper. The latter is also a difficult task as the grid structures are very fragile and even a small amount of mishandling easily breaks them. In contrast, with the carbon TEM grid we developed in this work, nano particle placement, in some cases, can be avoided, and surface modification for better nanoparticle attachment is also easily implemented. But even when using traditional placement techniques, such as dispersion casting, spraying, CVD, etc., the C-MEMS grids made here offer the advantage of adaptability in terms of size and precise structure. The water contact angle of the untreated C-MEMS carbon surface is around $79^{\circ}$, reported somewhere else. This surface does not require further treatment to make it more hydrophilic [30]. 
In our C-MEMS TEM grid fabrication process detailed below, we make the grid and the nanostructure at the same time. We use a polymer like SU8 for fabricating the supporting structures (the mesh structure like in a commercial grid) and place the nanofiber on it by EMS. In the pyrolysis process both the nanofiber and the TEM mesh structure are converted to carbon. After successful release of the carbon grids from the substrate, they are used directly in the TEM system, avoiding any further preparation step. Another major advantage of these carbon grids is that one can design them in a variety of sizes and configurations based on the requirements of the nanostructure one wants to study and the fabrication cost remains the same.

When working with biological samples TEM sample preparation becomes even more challenging as cells/tissues need normally to be dehydrated and fixed before being sectioned and then put onto grids. In this application, TEM grids that are bio-compatible are in high demand. Given the challenges described here, the authors do not claim that the proposed C-MEMS TEM grids will solve all the problems, however the glassy nature of the carbon TEM grids we have developed makes them suitable for biological applications. Cells/tissues can be grown on these grids or suspended CNWs and the complexity of sample preparation is reduced significantly $[14,31]$.

\section{C-MEMS TEM Grids}

The C-MEMS process as detailed above (see also Figure 1) was adapted for the fabrication of carbon TEM grids by adding an EMS fiber deposition step and etching the carbon structure with Buffered Oxide Etch (BOE), as seen in Figure 3. 


\subsection{Fabrication of SU8 grids}

For the fabrication of the SU8 grids two types of substrates were used: $\mathrm{Si} / \mathrm{SiO}_{2}$ wafers and bare $\mathrm{Si}$ wafers. The thickness of $\mathrm{SiO}_{2}$ on the $\mathrm{Si} / \mathrm{SiO}_{2}$ substrate was $1 \mu \mathrm{m}$ whereas there was only a native oxide layer on the Si substrates. SU8 2100 photoresist from Microchem® was spin coated to a thickness of $200 \mu \mathrm{m}$ on both substrates and this was followed by a softbake for 6 minutes at $65^{\circ} \mathrm{C}$ and then for 40 minutes at $95^{\circ} \mathrm{C}$. The prebake at $65^{\circ} \mathrm{C}$ was carried out in order to ensure a slower evaporation of the solvent resulting in a better coating and better adhesion to the substrate. The subsequent bake at $95{ }^{\circ} \mathrm{C}$ further densifies the SU8. The SU8 coated samples were then exposed to UV light through a photo mask and placed on a hot plate for 5 minutes at $65^{\circ} \mathrm{C}$ and for 14 minutes at $95^{\circ} \mathrm{C}$ for a Post Exposure Bake (PEB). The PEB increases the degree of cross-linking in the UV-exposed areas and makes the coating more resistant to solvents in the development step. After the PEB, the samples were dipped into the developer solution for 20 minutes and the solution was shaken for the entire time to ensure the complete removal of the un-exposed resist areas.

\subsection{Electromechanical Spinning (EMS) of Carbon Nanowires (CNWs)}

The fabricated SU8 TEM grid structures as described above were used as a substrate in which suspended polymer fibers were deposited using Electro Mechanical Spinning (EMS). For details on the EMS process consult our previous works [23,32]. In short, a voltage between 200 and $600 \mathrm{~V}$ is applied between a conductive needle (polymer/solvent dispenser) and a grounded substrate located at less than $1 \mathrm{~mm}$ from the needle tip. A droplet forms at the needle tip and upon poking the bubble with a needle -creating an artificial instability-a 
polymer jet initiates. In this way, nanofibers are precisely positioned on the SU8 TEM grids.

\subsection{Pyrolysis of the TEM Grids with Suspended Fibers}

The fabricated SU8 structures with the suspended polymer nanofibers were placed in a furnace for pyrolysis in a nitrogen environment. It is necessary to ensure the absence of even traces of oxygen, so the polymer is carbonized instead of burned. The furnace was heated from room temperature to $300^{\circ} \mathrm{C}$ at a ramp rate of $5^{\circ} \mathrm{C} / \mathrm{min}$ and was left for 1 hour at $300^{\circ} \mathrm{C}$ for temperature stabilization. Then the furnace temperature was ramped up to $900^{\circ} \mathrm{C}$ at $10^{\circ} \mathrm{C} / \mathrm{min}$. The samples were kept at $900^{\circ} \mathrm{C}$ for one hour before cooling down. The final step was to release the carbon TEM grid structures with the suspended CNWs from the substrate. For this purpose a BOE was used for etching the oxide layer from under the CMEMS TEM grid. We will see below that this last step is not always required as one can have self-releasing grids through stress management.

\section{Results and discussions}

In Figure 4a, we show SEM images (FEI Quanta 3D FEG) of a C-MEMS TEM grid with CNWs placed on it by EMS (magnified image is shown in Figure 4b). The substrate here was a $\mathrm{Si} / \mathrm{SiO}_{2}$ wafer and the C-MEMS TEM grid thickness was measured to be around $150 \mu \mathrm{m}$ using confocal microscope (Axion, Zeiss). We observed that grids of this thickness detached from the substrate surface after pyrolysis without the need of a release etch. We also observed that the grids were bent like convexo-concave structures and we measured the percentage of the grid diameter shrinkage, using SEM, and was found to be $45 \%$. 
With the lithographically patterned SU8 structure securely anchored to the substrate one expects that upon pyrolysis the shrinkage of the SU8 pattern would cause the square window openings to increase because of the tensile stress generated in the carbonizing film and thus stretch and thin the suspended polymer wires over these windows in the process. However, in the above experiments the square grid windows were reduced instead by $38 \%$. The original squares in the SU8 pattern were $150 \mu \mathrm{m} \times 150 \mu \mathrm{m}$.

To qualify our structure as a good TEM grid material we also investigated the CMEMS TEM grid's microstructure. Raman spectroscopy (Renishaw InVia Raman microscope) results of the C-MEMS TEM grids are presented in Figure 5. Two peaks centered around $1350 \mathrm{~cm}^{-1}$ and $1594 \mathrm{~cm}^{-1}$ corresponding to the D- and G-band, respectively are observed, typical for partial crystalline carbon with small crystal size. The intensity ratio $\left(\mathrm{I}_{\mathrm{D}} / \mathrm{I}_{\mathrm{G}}\right)$ of these bands, $\mathrm{D}$ - and G-band, defines the crystallinity in the structure; the lower the ratio is the higher the crystallinity and the lower the disordered nature of the carbon at hand [33]. From this results and our previous works [11] the microstructure of the carbon obtained here is glassy in nature.

The TEM image (FEI/Philips CM-20) of a $1.5 \mu \mathrm{m}$ diameter $\mathrm{CNW}$ is shown in Figure 6. Mostly disorder, glassy carbon structures are found at the edge of the CNW. Glassy carbon is highly chemical resistant and glassy carbon TEM grids could be used for nano samples that need further chemical micromachining for thinning while on the grid.

In Figure 7 we show SEM images for the same TEM grid geometry and thickness as in Figure 4 but on a bare Si substrate. In this case also, the grid structure peeled off after pyrolysis and the grid diameter and window dimension shrinkage were around $42 \%$ and 
$50 \%$ respectively. However in this case, the grid surface remained planar hinting towards a somewhat stronger attachment of the C-MEMS structure to the Si surface.

To avoid the release of the grids from the substrate through accumulated tensile stress - a difficult to control process - we made thinner SU8 structures and observed that thinner structures remain attached even onto a $\mathrm{Si} / \mathrm{SiO}_{2}$ substrate. In Figure 8, we show the SEM images of C-MEMS TEM grids carbonized from an initial SU8 grid thickness of $100 \mu \mathrm{m}$ on a $\mathrm{Si} / \mathrm{SiO}_{2}$ substrate. During the carbonization process the thickness of the SU8 shrinks to $60 \mu \mathrm{m}$ and the wire diameter to $180 \mathrm{~nm}$. Also as the C-MEMS grids remained attached to the substrate, the window sizes now expanded (by about 30\%) as we had expected. In the case of thicker SU8 structures, the accumulated stress releases the grids from the substrate and as they are not anchored to the substrate anymore the film and the windows in it contract instead of expanding. Using buffered oxide etch (BOE) the CMEMS grids were released from the substrate with the suspended CNWs firmly attached and ready for TEM analysis.

This window expansion causes the suspended polymer fiber to thin and the resulting CNWs are thus also thinner than when using thicker grids that peel off. As demonstrated elsewhere by our team [34], thinner CNWs derived from a more stretched polymer wire are more graphitic due to the pre-alignment of the polymer chains in the wire [35]. We have reconfirmed this finding using the C-MEMS TEM grid.

Carbon TEM grids and the CNWs fabricated on them with EMS were inserted into a TEM without any additional sample preparation. TEM image and diffraction pattern of the CNW are shown in Figure 9. The ring shape diffraction pattern indicates a short-range 
crystalline order, and the foggy pattern surrounded by the ring pattern is indicative of defects in the graphitic phase.

The TEM carbon grids we developed here for the study of suspended CNWs may be used for many other nano-structured materials and many in-situ TEM experiments are enabled by it. For example, growth of CNTs can be realized directly on the carbon grid with the grid itself acting as the source of carbon [36].

\section{Conclusions}

In general, nanoparticles, CNTs and nanowires are synthesized/fabricated and usually placed on conventional TEM grids for characterization. However, this strategy is not suitable for characterization of some C-MEMS/C-NEMS structures, like suspended carbon nanofibers. These are fabricated in a two-step process. First, the fibers are deposited over posts by electrospinning and then converted into carbon by pyrolysis in an inert environment. Even if polymer nanofibers could be electrospun on conventional TEM grids, these grids are not suitable for high temperature processes like pyrolysis. Additionally, geometry and material of grid over which fibers are deposited are relevant to the final micro/nanostructure of the pyrolyzed fibers. Being able to characterize fibers that have been suspended over polymer grid and then pyrolyzed is very important. In this paper, we have presented a fabrication method of carbon grids using a $\mathrm{Si} / \mathrm{SiO}_{2}$ substrate. In this method, suspending grid and suspended fibers are pyrolyzed at the same time. It is found that the grid is peeled off from substrate when the thickness of the SU8 grid structure, before pyrolysis, is more than $200 \mu \mathrm{m}$. It is shrunk around $45 \%$ in diameter and also bent in shape after pyrolysis. The same grid is fabricated on Si substrate using the same fabrication 
process flow to investigate the reason of peel off. We have also observed the same grid peel off from the substrate, but the shape is planer in that case. However, when the SU8 grid thickness is around $100 \mu \mathrm{m}$ and fabricated on $\mathrm{Si} / \mathrm{SiO}_{2}$, it does not peel off and one more step of $\mathrm{BOE}$ is needed to release the grid from substrate. It is a big advantage that one can design the grid pattern based on requirements as the polymer (SU8) used in the grid fabrication is compatible with photolithography. The low cost carbon grids could be very useful for characterization of nanoparticles, CNTs and other synthesized materials replacing conventional costly grids. Also these grids have the potential to be used directly as the substrates for the growth of CNTs and other nanostructures.

\section{Acknowledgement}

B. Pramanick and A. Salazar equally contributed to this work. The authors would like to acknowledge the financial support from Conacyt in Mexico (grant no. CB-2014-1-241458 and postdoctoral fellowship no. 232499); and from NSF in USA (grant no. 1449397). This work was also financially supported by the Sensors and Devices Group of Tecnologico de Monterrey. Raman spectroscopy was performed at Tecnologico de Monterrey water center Facility. TEM and SEM works were performed at the UC Irvine Materials Research Institute (IMRI) using instrumentation funded in part by the National Science Foundation Center for Chemistry at the Space-Time Limit (CHE-082913).

\section{References:}

[1] A.K. Wanekaya, W. Chen, N.V. Myung, A. Mulchandani, Nanowire-Based Electrochemical Biosensors, Electroanalysis. 18(6) (2006) 533-550. doi:10.1002/elan.200503449.

[2] C. Wang, M.J. Madou, From MEMS to NEMS with carbon, Biosens. Bioelectron. 20(10) (2005) 2181-2187. doi:10.1016/j.bios.2004.09.034.

[3] J. Huang, Q. Wan, Gas Sensors Based on Semiconducting Metal Oxide OneDimensional Nanostructures, Sensors. 9(12) (2009) 9903-9924. doi:10.3390/s91209903. 
[4] C. Berger, Z. Song, T. Li, X. Li, A.Y. Ogbazghi, R. Feng, Z. Dai, A.N. Marchenkov, E.H. Conrad, P.N. First, W.A. de Heer, Ultrathin Epitaxial Graphite: 2D Electron Gas Properties and a Route toward Graphene-based Nanoelectronics, J. Phys. Chem. B. 108(52) (2004) 19912-19916. doi:10.1021/jp040650f.

[5] C. Wang, L. Taherabadi, G. Jia, M. Madou, Y. Yeh, B. Dunn, C-MEMS for the Manufacture of 3D Microbatteries, Electrochem. Solid-State Lett. 7(11) (2004) A435-A438. doi:10.1149/1.1798151.

[6] C. Wang, G. Jia, L.H. Taherabadi, M.J. Madou, A novel method for the fabrication of high-aspect ratio C-MEMS structures, J. Microelectromechanical Syst. 14(2) (2005) 348-358. doi:10.1109/JMEMS.2004.839312.

[7] M. Irie, S. Endo, C.L. Wang, T. Ito, Fabrication and properties of lateral p-i-p structures using single-crystalline CVD diamond layers for high electric field applications, Diam. Relat. Mater. 12(2) (2003) 1563-1568. doi:10.1016/S09259635(03)00243-7.

[8] B.K. Tay, D. Sheeja, L.J. Yu, On stress reduction of tetrahedral amorphous carbon films for moving mechanical assemblies, Diam. Relat. Mater. 12(2) (2003) 185-194. doi:10.1016/S0925-9635(03)00021-9.

[9] B.Y. Park, L. Taherabadi, C. Wang, J. Zoval, M.J. Madou, Electrical Properties and Shrinkage of Carbonized Photoresist Films and the Implications for Carbon Microelectromechanical Systems Devices in Conductive Media, J. Electrochem. Soc. 152(12) (2005) J136-J143. doi:10.1149/1.2116707.

[10] M.J. Madou, From MEMS to Bio-MEMS and Bio-NEMS: Manufacturing Techniques and Applications, 3rd ed. edition, CRC Press, (2011). ISBN-10: 142005516X

[11] S. Ranganathan, R. McCreery, S.M. Majji, M. Madou, Photoresist-Derived Carbon for Microelectromechanical Systems and Electrochemical Applications, J. Electrochem. Soc. 147(1) (2000) 277-282. doi:10.1149/1.1393188.

[12] R.R. Kamath, M.J. Madou, Three-Dimensional Carbon Interdigitated Electrode Arrays for Redox-Amplification, Anal. Chem. 86(6) (2014) 2963-2971. doi:10.1021/ac4033356.

[13] A. Mardegan, R. Kamath, S. Sharma, P. Scopece, P. Ugo, M. Madou, Optimization of Carbon Electrodes Derived from Epoxy-based Photoresist, J. Electrochem. Soc. 160(8) (2013) B132-B137. doi:10.1149/2.107308jes.

[14] S. Holmberg, M. Rodriguez-Delgado, R.D. Milton, N. Ornelas-Soto, S.D. Minteer, R. Parra, M.J. Madou, Bioelectrochemical Study of Thermostable Pycnoporus sanguineus CS43 Laccase Bioelectrodes Based on Pyrolytic Carbon Nanofibers for Bioelectrocatalytic O2 Reduction, ACS Catal. 5(12) (2015) 7507-7518. doi:10.1021/acscatal.5b01600.

[15] R. Martinez-Duarte, P. Renaud, M.J. Madou, A novel approach to dielectrophoresis using carbon electrodes, Electrophoresis. 32(17) (2011) 2385-2392. doi:10.1002/elps.201100059.

[16] C. Kim, Y.-O. Choi, W.-J. Lee, K.-S. Yang, Supercapacitor performances of activated carbon fiber webs prepared by electrospinning of PMDA-ODA poly(amic acid) solutions, Electrochimica Acta. 50(2-3) (2004) 883-887. doi:10.1016/j.electacta.2004.02.072.

[17] M. Blazewicz, Carbon materials in the treatment of soft and hard tissue injuries, Eur. Cell. Mater. 2(2) (2001) 21-29. ISSN 1473-2262 
[18] S. Sharma, Microstructural Tuning of Glassy Carbon for Electrical and Electrochemical Sensor Applications, PhD Thesis, University of California Irvine, (2013).

[19] Y. Lim, J.-I. Heo, H. Shin, Suspended Carbon Nanowire-Based Structures for Sensor Platforms, ECS Trans. 61(7) (2014) 25-29. doi:10.1149/06107.0025ecst.

[20] A.V. Melechko, V.I. Merkulov, T.E. McKnight, M.A. Guillorn, K.L. Klein, D.H. Lowndes, M.L. Simpson, Vertically aligned carbon nanofibers and related structures: Controlled synthesis and directed assembly, J. Appl. Phys. 97(4) (2005) 41301. doi:10.1063/1.1857591.

[21] T. Saito, T. Yamada, D. Fabris, H. Kitsuki, P. Wilhite, M. Suzuki, C.Y. Yang, Improved contact for thermal and electrical transport in carbon nanofiber interconnects, Appl. Phys. Lett. 93(10) (2008) 102108. doi:10.1063/1.2979710.

[22] S. Sharma, M. Madou, A new approach to gas sensing with nanotechnology, Philos. Trans. R. Soc. Lond. Math. Phys. Eng. Sci. 370(1967) (2012) 2448-2473. doi:10.1098/rsta.2011.0506.

[23] G. Canton, T. Do, L. Kulinsky, M. Madou, Improved conductivity of suspended carbon fibers through integration of C-MEMS and Electro-Mechanical Spinning technologies, Carbon. 71 (2014) 338-342. doi:10.1016/j.carbon.2014.01.009.

[24] H. Lorenz, M. Despont, N. Fahrni, N. LaBianca, P. Renaud, and P. Vettiger, SU-8: A Low-Cost Negative Resist for MEMS, J. Micromech. Microeng. 7(3) (1997) 121124. doi:10.1088/0960-1317/7/3/010.

[25] C. Liu, Y. Liu, M. Sokuler, D. Fell, S. Keller, A. Boisen, H.J. Butt, G.K. Auernhammer, E. Bonaccurso, Diffusion of water into SU-8 microcantilevers, Phys. Chem. Chem. Phys. 12(2010) 10577-10583. doi:10.1039/c002478c.

[26] S.M. Mukhopadhyay, Sample Preparation for Microscopic and Spectroscopic Characterization of Solid Surfaces and Films, in: S. Mitra (Ed.), Sample Prep. Tech. Anal. Chem., Wiley-Interscience, (2003) 377-411. ISBN 978-0-471-45781-7

[27] J.E. Bonevich, W.K. Haller, Measuring the Size of Nanoparticles Using Transmission Electron Microscopy (TEM), NIST-NCL Jt. Assay Protoc. PCC-7 Version 1. (2010) $1-13$.

[28] B. Michen, C. Geers, D. Vanhecke, C. Endes, B. Rothen-Rutishauser, S. Balog, A. Petri-Fink, Avoiding drying-artifacts in transmission electron microscopy: Characterizing the size and colloidal state of nanoparticles, Sci. Rep. 5(9793) (2015) 1-7. doi:10.1038/srep09793.

[29] U. Aebi, T.D. Pollard, A glow discharge unit to render electron microscope grids and other surfaces hydrophilic, J. Elec. Microscopy Technique. 7 (1987) 29-33.

[30] D. Kim, B. Pramanick, A. Salazar, I-W. Tcho, M.J. Madou, E.S. Jung, Y-K. Choi, H. Hwang, 3D carbon electrode based triboelectric nanogenerator, Adv. Mater. Technol. 1600160 (2016). doi:10.1002/admt.201600160.

[31] M. Huber, P. Heiduschka, S. Kienle, C. Pavlidis, J. Mack, T. Walk, G. Jung, S. Thanos, Modification of glassy carbon surfaces with synthetic laminin-derived peptides for nerve cell attachment and neurite growth, J. Biomed. Mater. Res. 41(2) (1998) 278-288. doi:10.1002/(SICI)1097-4636(199808)41:2<278::AIDJBM13>3.0.CO;2-H.

[32] G.S. Bisht, G. Canton, A. Mirsepassi, L. Kulinsky, S. Oh, D. Dunn-Rankin, M.J. Madou, Controlled Continuous Patterning of Polymeric Nanofibers on Three- 
Dimensional Substrates Using Low-Voltage Near-Field Electrospinning, Nano Lett. 11(4) (2011) 1831-1837. doi:10.1021/nl2006164.

[33] A.C. Ferrari, J. Robertson, Interpretation of Raman spectra of disordered and amorphous carbon, Phys. Rev. B. 61(20) (2000) 14095-14107. doi:10.1103/PhysRevB.61.14095.

[34] G. Canton, Development of Electro-Mechanical Spinning for Controlled Deposition of Carbon Nanofibers, PhD Thesis, University of California Irvine, 2014.

[35] Y. Ji, C. Li, G. Wang, J. Koo, S. Ge, B. Li, J. Jiang, B. Herzberg, T. Klein, S. Chen, J.C. Sokolov, M.H. Rafailovich, Confinement-induced super strong PS/MWNT composite nanofibers, EPL Europhys. Lett. 84(5) (2008) 56002. doi:10.1209/02955075/84/56002.

[36] N. Petkov, In Situ Real-Time TEM Reveals Growth, Transformation and Function in One-Dimensional Nanoscale Materials: From a Nanotechnology Perspective, Int. Sch. Res. Not. 2013 (2013) 1-21. doi:10.1155/2013/893060. 


\section{Figures}

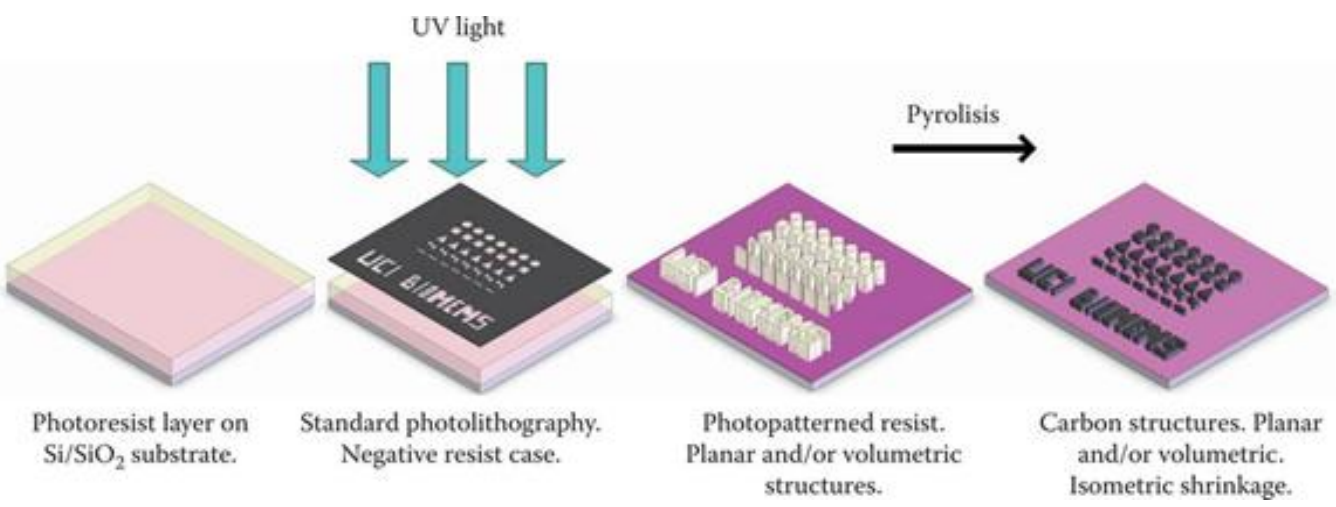

Figure 1: An illustration of one type of carbon-based microelectromechanical system (CMEMS): photolithographic patterning of a polymer precursor (SU8 photo resist) and pyrolysis [10].
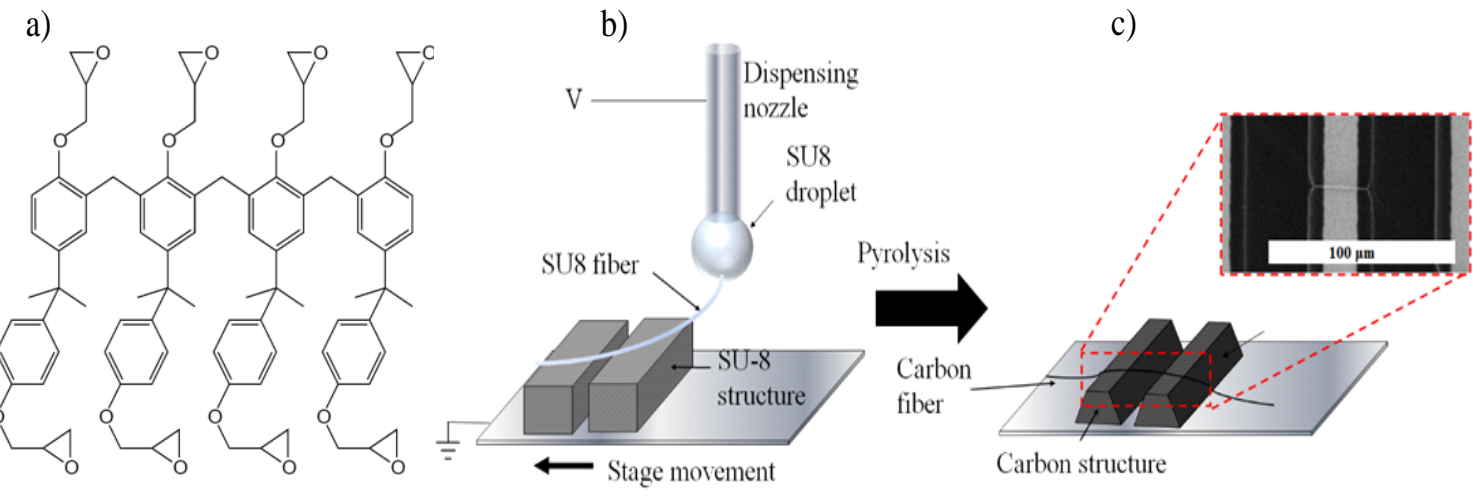

Figure 2. a) Chemical structure of SU8. b) EMS deposition of SU8 fibers over photopatterned SU8 structures. c) Monolithic carbon structure obtained after pyrolysis

[25]. 
(a) Spin-coat of photoresist

(b) UV-exposure
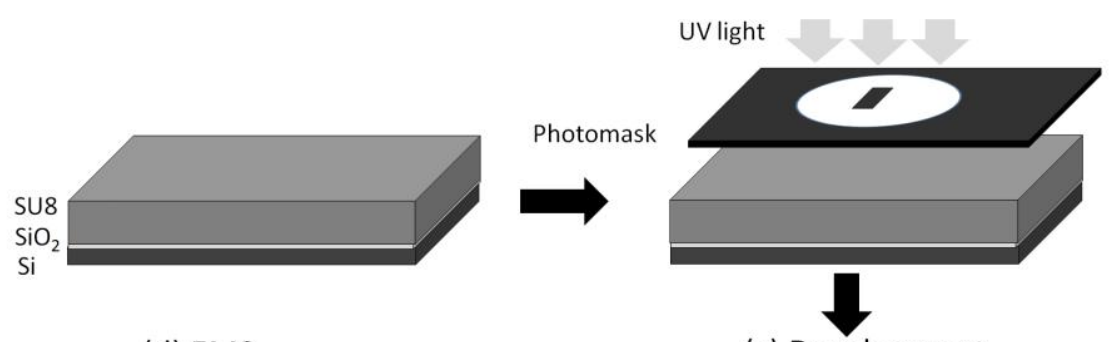

(d) EMS
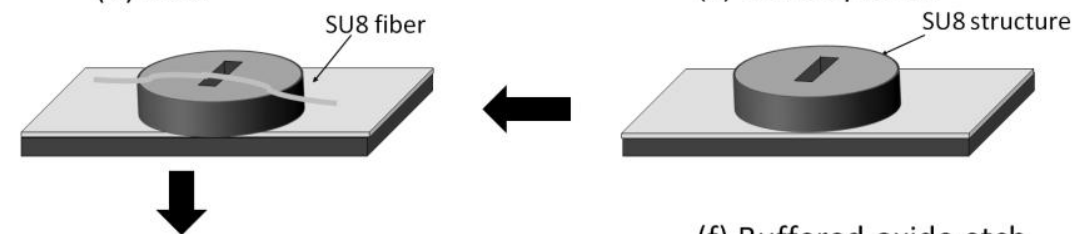

(e) Pyrolysis
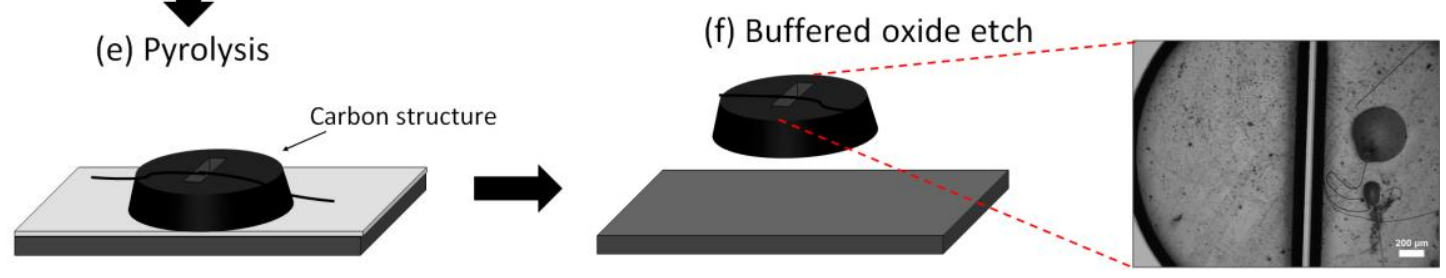

Figure 3. Fabrication process steps of carbon TEM Grid. Steps (d) EMS and (f) Buffered oxide etch are added to the conventional C-MEMS process.
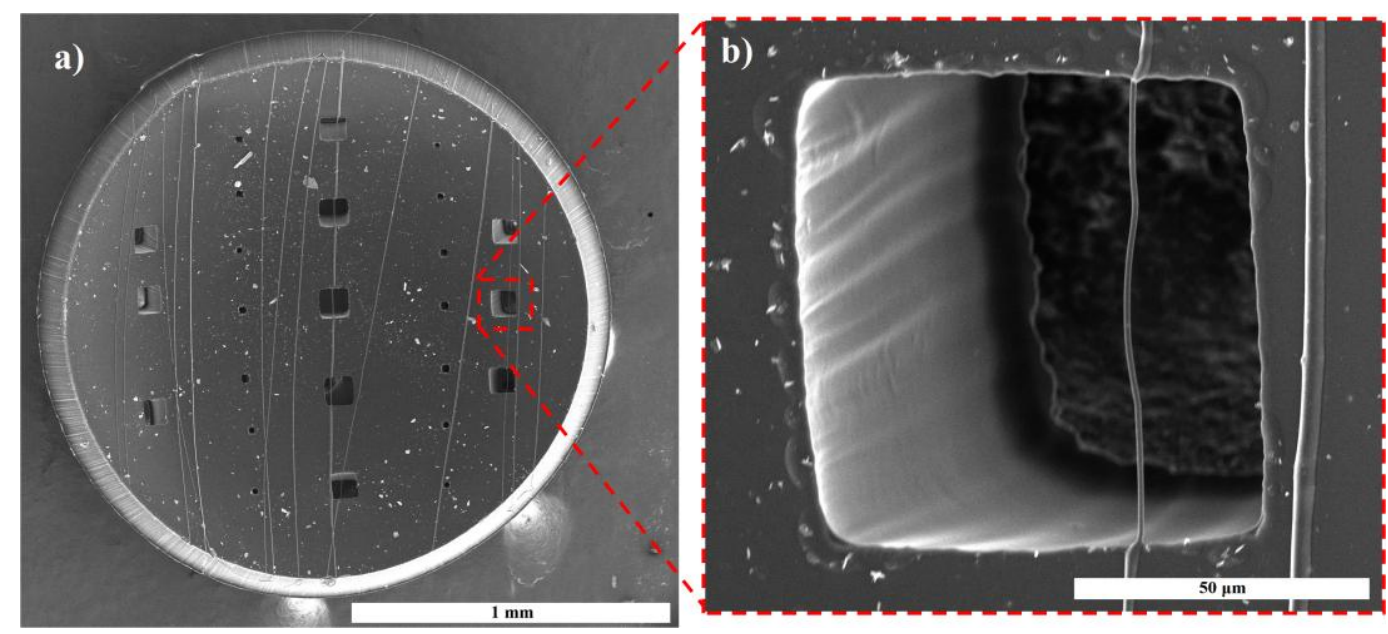

Figure 4. SEM images of: a) Carbon TEM grids on a $\mathrm{Si} / \mathrm{SiO}_{2}$ substrate. b) Magnified view of suspended $\mathrm{CNW}$. 
Raman Shift vs Intensity

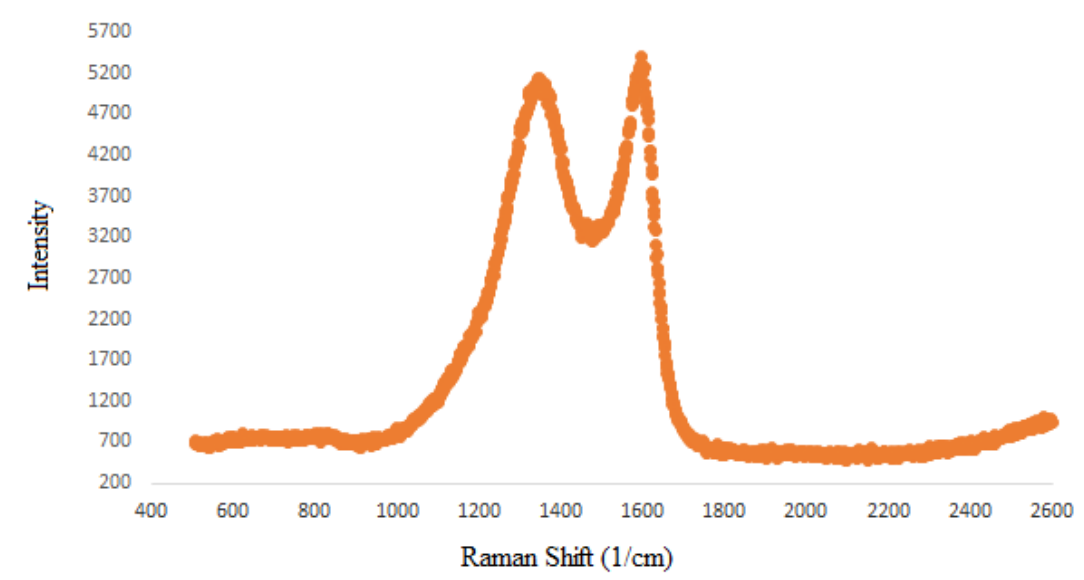

Figure 5. Raman spectroscopy result of the TEM grid.

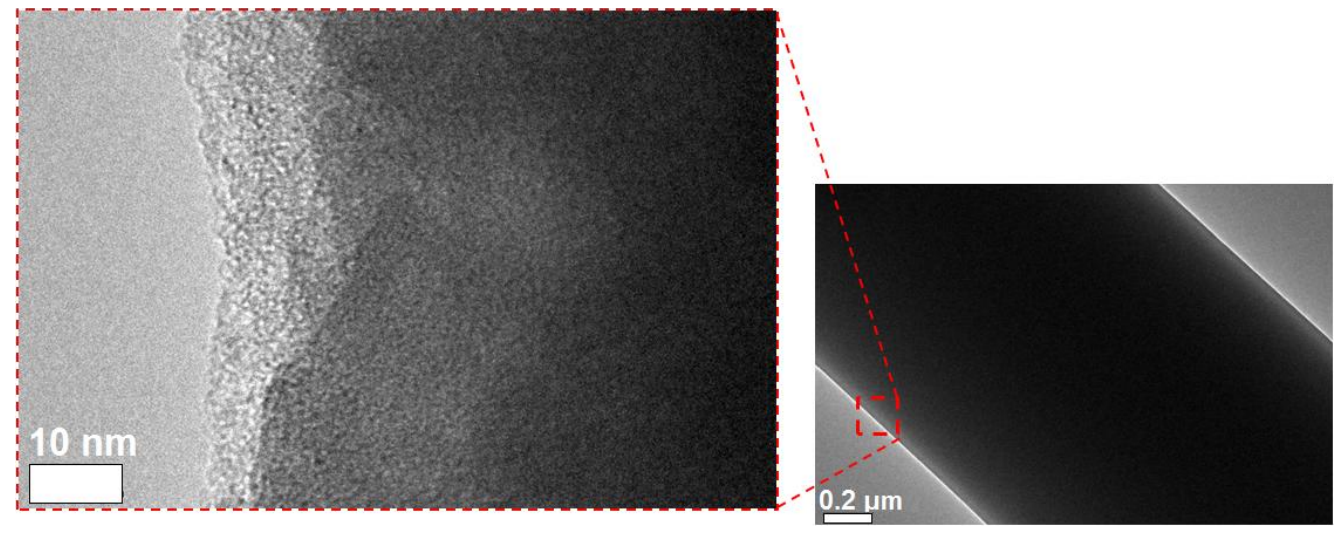

Figure 6. TEM image of suspended CNW of $1.5 \mu \mathrm{m}$ diameter.
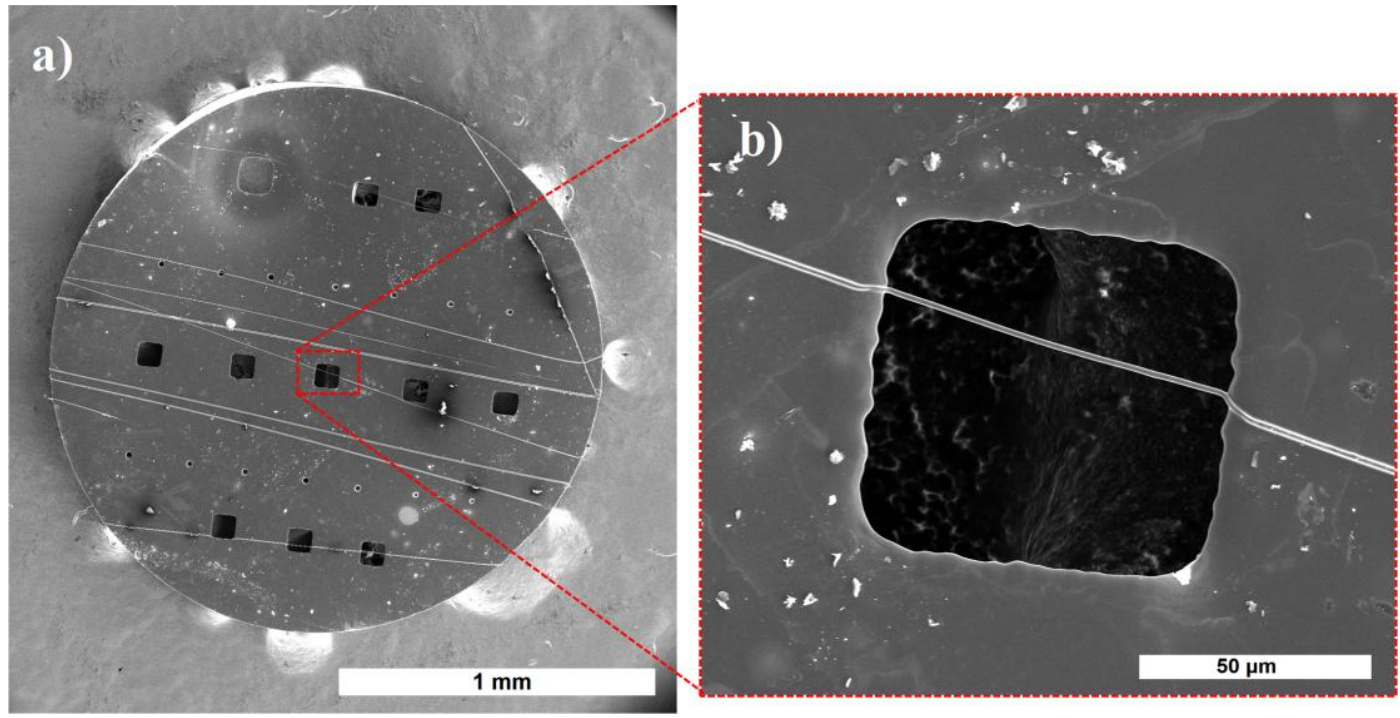

Figure 7. SEM images of: a) Carbon TEM grids on a Si substrate. b) Magnified view of suspended CNW. 

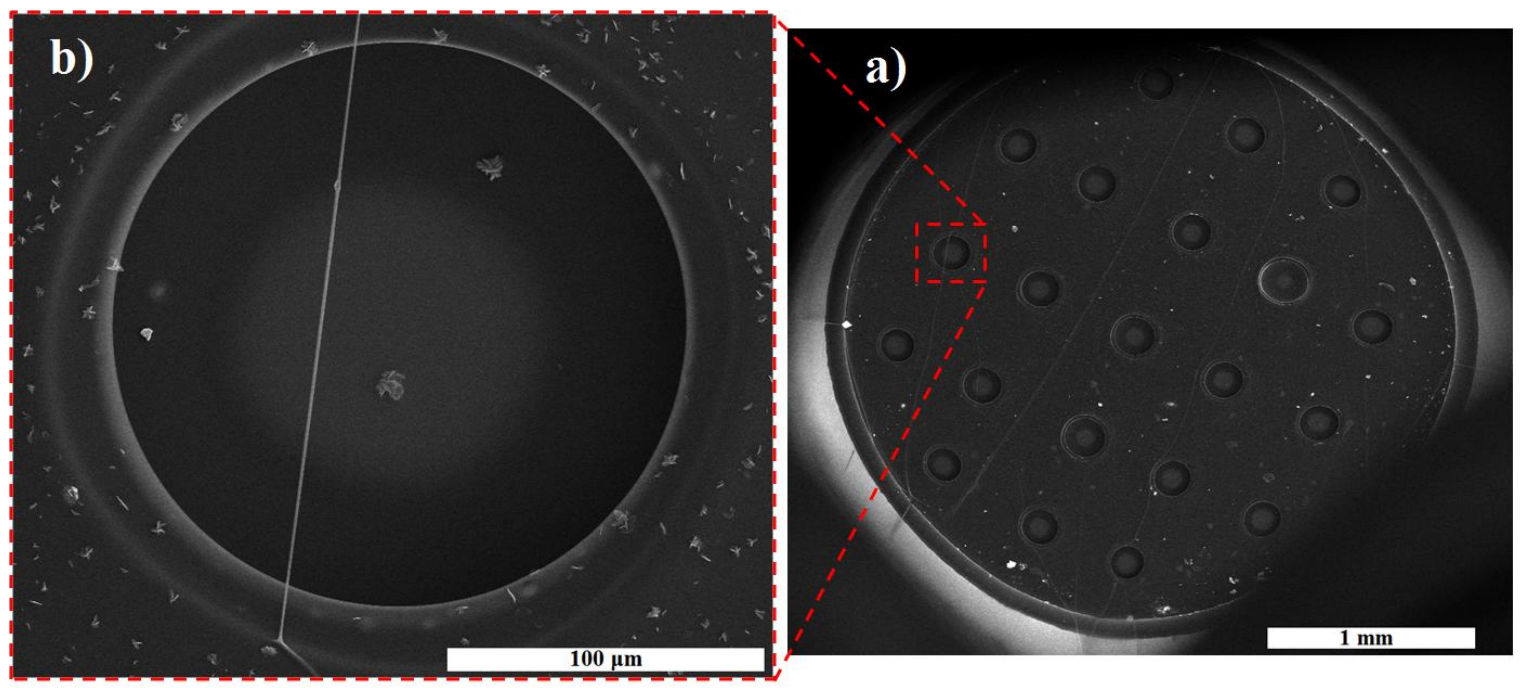

Figure 8. SEM images of $100 \mu \mathrm{m}$ thick: a) Carbon TEM grids on a $\mathrm{Si} / \mathrm{SiO} 2$ substrate. b) Magnified view of suspended CNW.
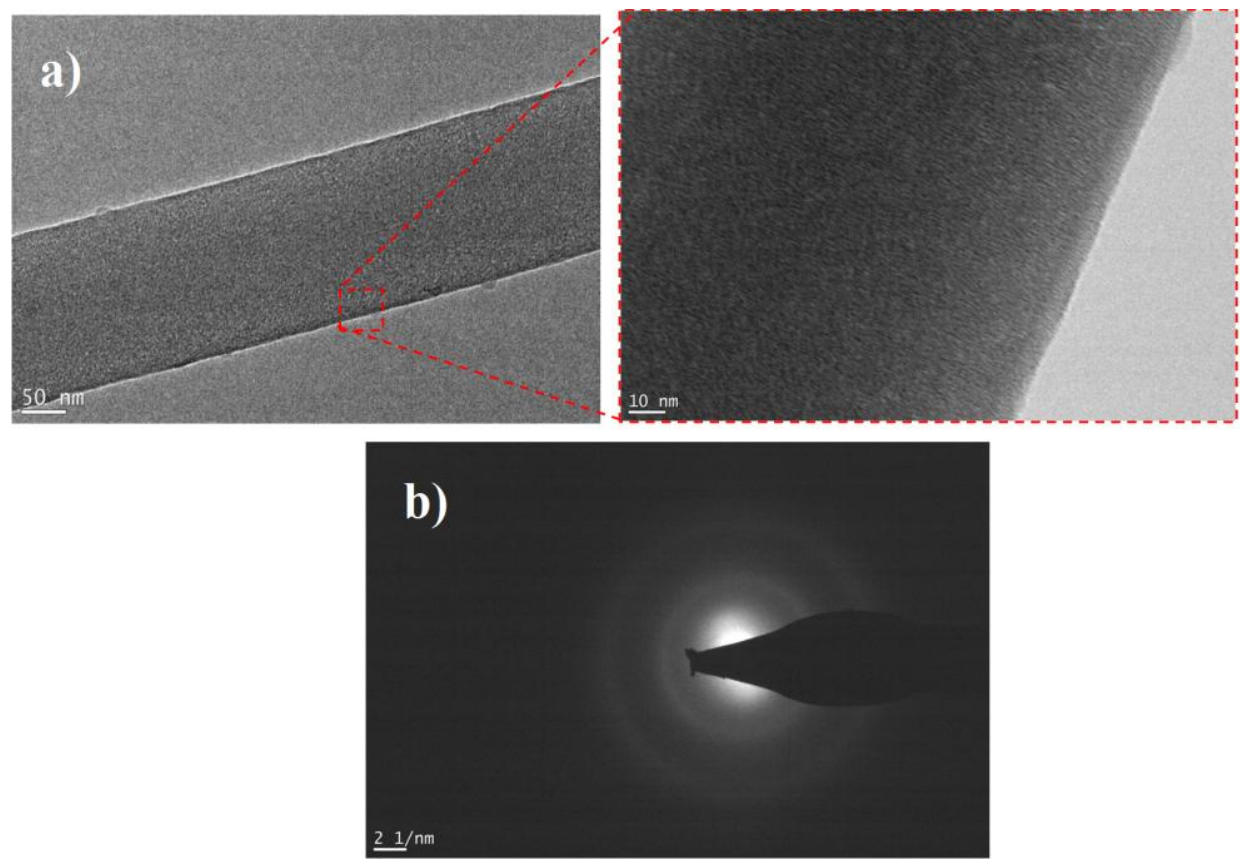

Figure 9. a) TEM image of suspended CNW of $180 \mathrm{~nm}$ in diameter (inset shows a magnified view of the edge of the CNW). b) Diffraction pattern of 180 nm diameter $\mathrm{CNW}$. 


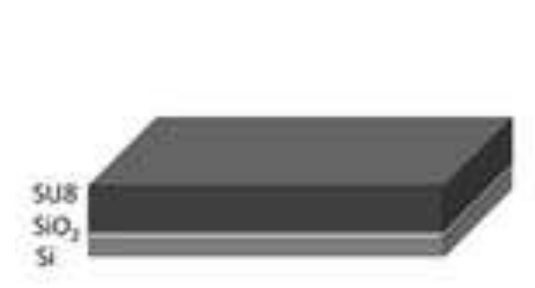

(d) EMS

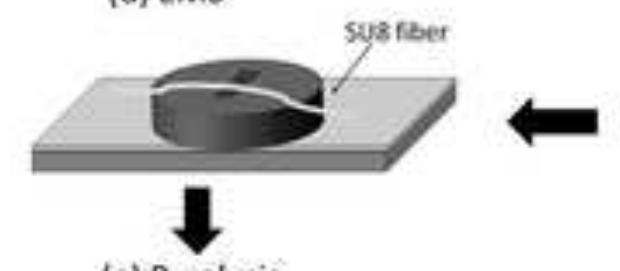

(e) Pyrolysis

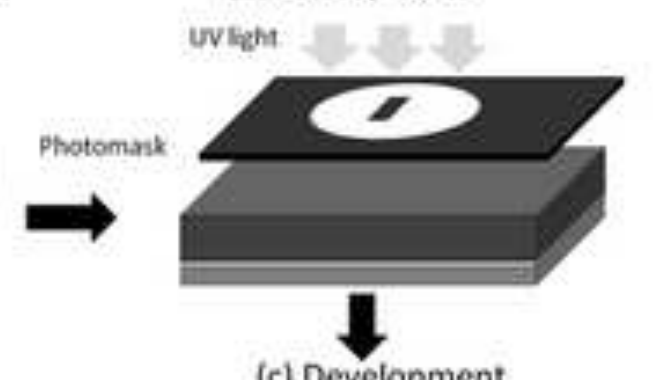

(c) Development

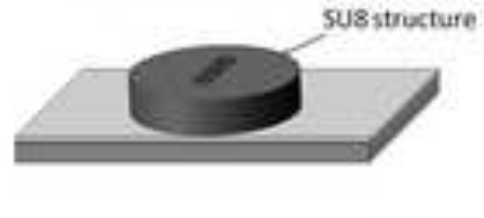

(f) Buffered oxide etch

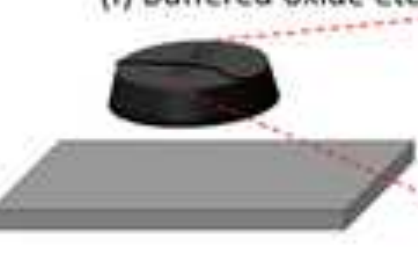

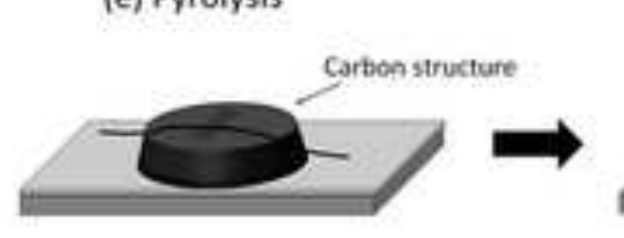
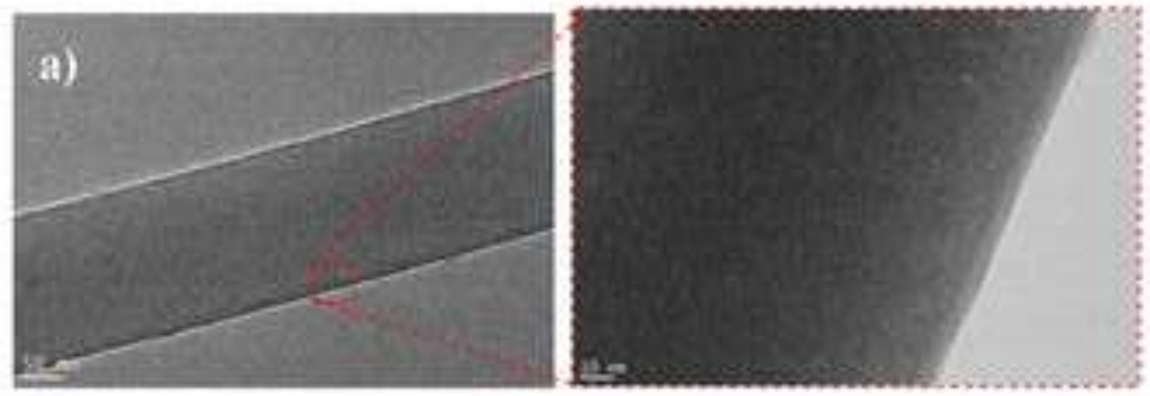

b)
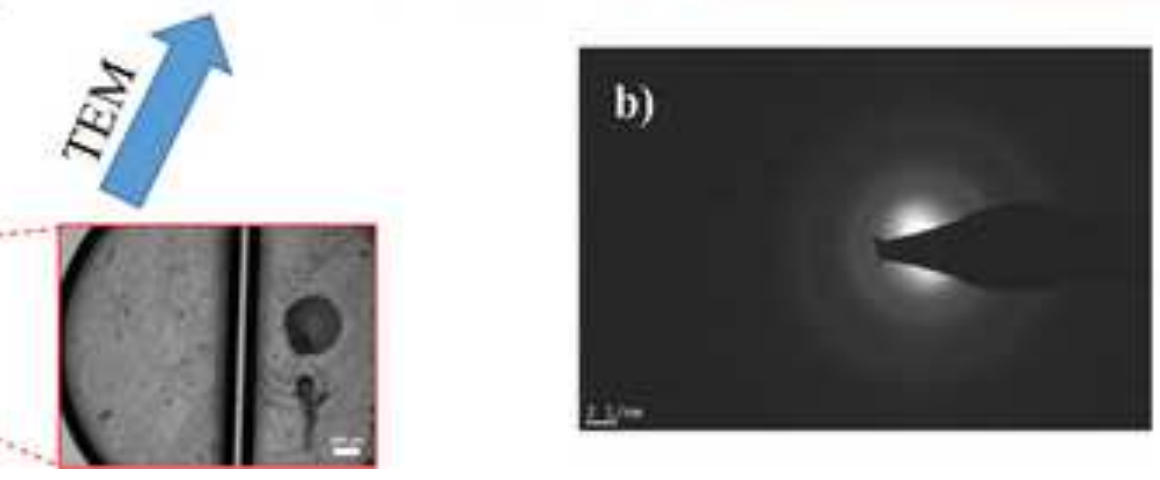